\title{
Isolated Renal Hydatid Cyst Misdiagnosed and Operated as a Cystic Renal Tumor
}

\author{
Rabea A. Gadelkareem ${ }^{a}$ Ayman A. Elqady ${ }^{a}$ Sayed K. Abd-Elshafy ${ }^{b}$ \\ Hisham Imam ${ }^{c}$ Hassan A. Abolella ${ }^{a}$ \\ ${ }^{a}$ Assiut Urology and Nephrology Hospital, Faculty of Medicine, Assiut University, Assiut, Egypt; bepartment of \\ Anesthesia and Intensive Care, Assiut University Hospital, Faculty of Medicine, Assiut University, Assiut, Egypt; \\ 'Department of Diagnostic Radiology, Assiut University Hospital, Faculty of Medicine, Assiut University, Assiut, Egypt
}

\section{Significance of the Study}

- Solitary single-cavity renal hydatid cysts with a "double-contour" appearance have been rarely described and may warrant studying. Misdiagnosis of the lesion as a renal tumor indicated radical nephrectomy with retrograde diagnosis. Isolated renal hydatid cyst should be considered during differential diagnosis of renal cystic lesions.

\section{Keywords}

Renal hydatid cyst · Cystic renal tumor · Echinococcus granulosus · Renal pseudotumor

\begin{abstract}
Objective: The aim of this work is the presentation of a case of isolated renal hydatid cyst with novel findings and an unusual surgical scenario. Clinical Presentation and Intervention: A 54-year-old female patient presented with left loin pain and a palpable left renal mass. Imaging described a well-demarcated left renal cystic lesion with a double-layer wall. Radical nephrectomy was performed due to the possibility of malignancy. On retrograde revision, the double-layer wall represented the detached germinative membrane of a hydatid cyst that was confirmed by histopathology. Conclusion: Isolated renal hydatid cyst could be misinterpreted as a renal tumor. It should be considered in the differential diagnosis of renal cystic lesions.

(c) 2018 The Author(s)

Published by S. Karger AG, Basel
\end{abstract}

\section{KARGER}

E-Mail karger@karger.com www.karger.com/mpp

\section{Introduction}

Hydatid disease is a worldwide zoonosis due to a parasitic infestation usually caused by Echinococcus granulosus. However, urogenital hydatid disease is a rare pathology comprising only $2-3 \%$ of all cases $[1,2]$. Urogenital sites rank third after the liver and lungs, which are the main predilection sites in more than $90 \%$ of cases. Although renal hydatid cyst is the most common form of urogenital hydatidosis, its isolated presentation is an extremely rare clinical entity [3], and it may be misinterpreted as a renal tumor $[1,4]$.

\section{Case Report}

A 54-year-old Egyptian female patient from an urban area presented with left loin pain of 14 months' duration. Physical examination revealed an average body build and normal vital signs. There was a nontender, fairly palpable left-sided renal mass.

Rabea Ahmed Gadelkareem

Assiut University

Elgamaa Street

Assiut 71515 (Egypt)

E-Mail dr.rabeagad@yahoo.com 
Abdominal ultrasound showed a cystic lesion occupying the lower and mid zones of the left kidney with internal septa, a thickened irregular wall, and turbid contents that looked like soft tissues. Noncontrast computed tomography was performed before presentation of the patient to our center and described a complex cystic mass occupying most of the left kidney with a well-defined outline, thickened wall, and a suggestion of a soft tissue compartment. Its dimensions were $10.5 \times 7.0 \times 5.5 \mathrm{~cm}$ with a hypodense appearance. The thickened wall was double layered in most of its circumference (Fig. 1). Magnetic resonance imaging, scheduled as a complementary study, showed similar findings to computed tomography. Moreover, a diffuse-weighted study failed to prove or exclude the suspicion of malignancy. It suggested an intra-cyst hemorrhage (Fig. 2a, b). Whole body bones, the chest, and other abdominal organs were shown to be free of expected metastases by imaging.

A urine analysis, renal function tests, and routine tests were unremarkable with eosinophilia. Owing to the suspicion of malignancy, open radical nephrectomy was performed through a left subcostal incision. Extensive adhesions were encountered with difficulties in dissection of the mass from the vicinities of the pancreas and spleen due to extensive tissue reactions. However, the operation was completed without rupture in $2 \mathrm{~h}$ and $25 \mathrm{~min}$. Blood loss was $250 \mathrm{~mL}$, necessitating the transfusion of 1 unit of blood. The postoperative course was uneventful.

The cut-surface of the mass showed a true and well-developed unilocular cyst involving the major parts of renal parenchyma and lined by a whitish membrane detached from the surrounding walls (Fig. 3). In retrograde revision, the lesion was a renal hydatid cyst and its detached germinative membrane with the reactive capsule was responsible for the thickened double-layer wall appearance. Microscopic and histopathological examinations clearly described and confirmed the diagnosis of renal hydatid cyst (Fig. 4a-d) and excluded malignancy. Clear gross and histopathological pictures reduced the necessity of postoperative serological testing for echinococcosis. However, a course of albendazole, $400 \mathrm{mg}$ twice daily, was prescribed for 4 weeks to reduce the possibilities of a relapse.

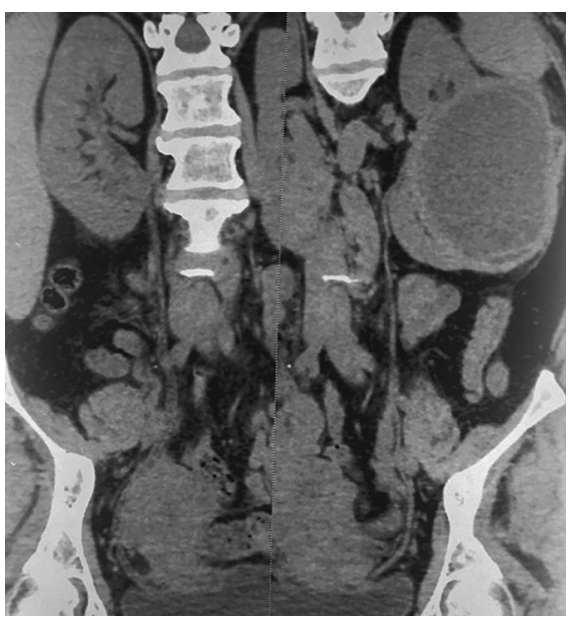

Fig. 1. Abdominal computed tomography coronal view: a unilocular cyst involving major parts of the left kidney with a double-contour appearance.

\section{Discussion}

Hydatid disease is a worldwide parasitic infestation transmitted from animals like dogs, which are the primary hosts, to other animals, such as sheep and cattle as the intermediate hosts, to humans. Transmission to humans occurs by ingestion of contaminated soils or vegetables [4]. The current case did not have a clear history of contact with animals. Thus, infestation may have occurred via contaminated foods.

Although it has no specific common clinical presentation, renal hydatid cyst has a suggestive characteristic appearance on imaging, surgical cut-surface, and histopathological examination [4]. The combination of a relevant clinical history, imaging tools, and laboratory investiga-
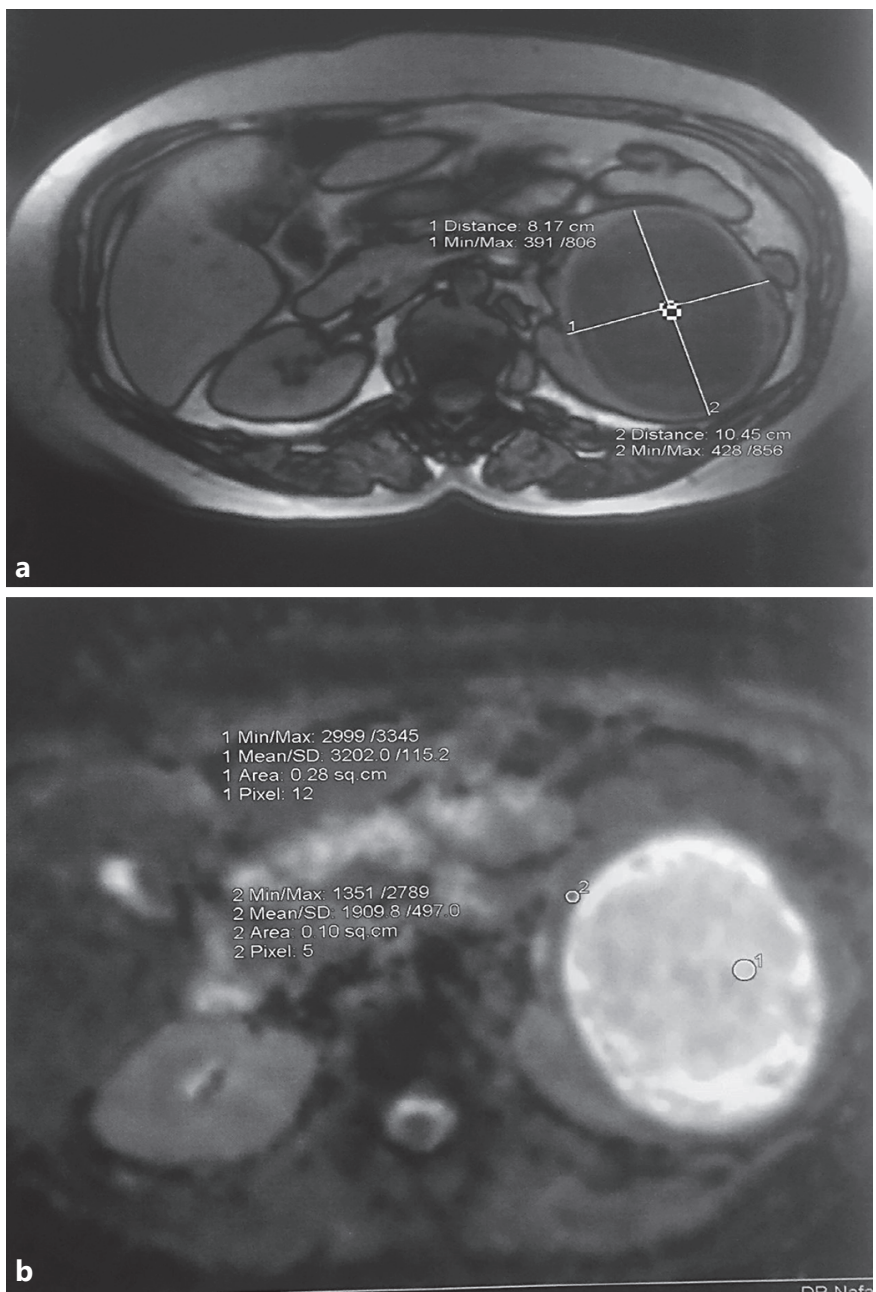

Fig. 2. Magnetic resonance imaging of the abdomen. a Transverse view showing the large cyst occupying great parts of the left kidney with a double-layer wall. b Diffuse-weighted study showing internal signals that were misinterpreted as intramural hemorrhage. 
tions provides a reliable preoperative diagnosis in only half of all cases.

On retrograde revision of the imaging studies in the current case, a well-demarcated unilocular renal cyst was observed. The detached germinative membrane was a double-layered membrane with the cyst wall, which we refer to as a "double-contour" appearance. This appearance has been previously described as a "water-lily sign." Cystic echinococcosis lesions have been classified by the World Health Organization into 5 grades. Grade 1 and 2 represent the early stage of disease where the production of scolices is active. Grade 3 is a transitional stage that represents the start of degeneration of the cyst with detachment of the germinal membrane from the cyst wall. It has been differentiated into grade $3 \mathrm{a}$ for a solitary cyst with multiple septa and grade $3 \mathrm{~b}$ for multiple small cysts in solid parenchymal tissues. Grades 4 and 5 refer to old and degenerative stages of the disease with calcification. Based on this classification, imaging findings of the current case refer to a grade $3 a$ hydatid cyst (G3a) [5-7]. In the current case, this finding was confirmed by the cutsurface of the mass after nephrectomy. A typical radio-

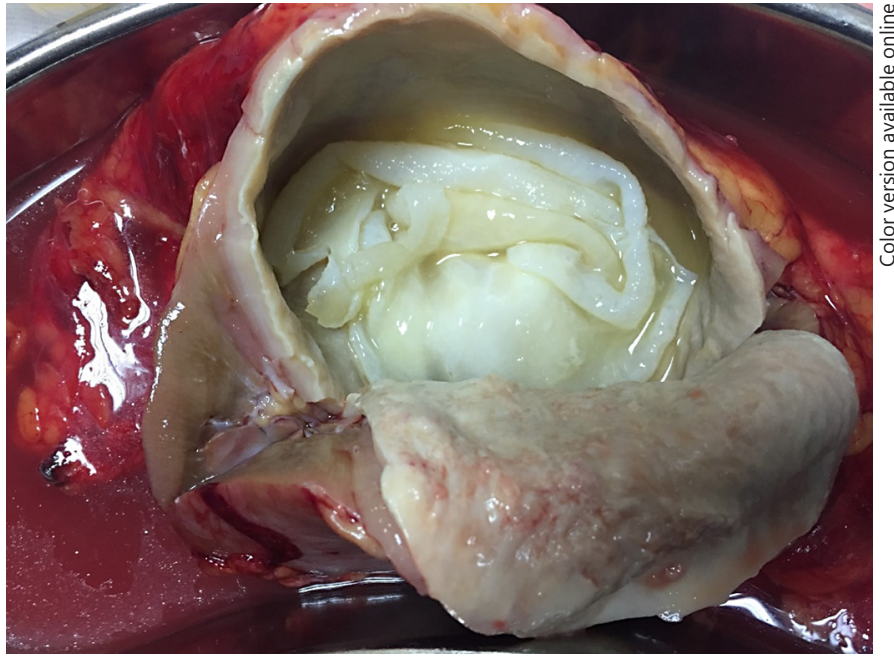

Fig. 3. Cut-surface of the left kidney showing a well-formed huge unilocular cyst filled with a detached folded whitish germinative membrane.
Fig. 4. Histopathological overview of the renal hydatid cyst. a Low-power view of the histology of inner scolices forming and the thick outer layer of the hydatid cyst. HE stain. Original magnification $\times 40$. b Histology of the wall of the hydatid cyst with a characteristic chitinous layer admixed with inflammatory cells. HE stain. Original magnification $\times 100$. c High-power view of layers of hydatid cyst admixed with plenty of eosinophils. HE stain. Original magnification $\times 400$. d Kidney parenchyma adjacent to the hydatid cyst exhibits chronic pyelonephritis. HE stain. Original magnification $\times 100$.
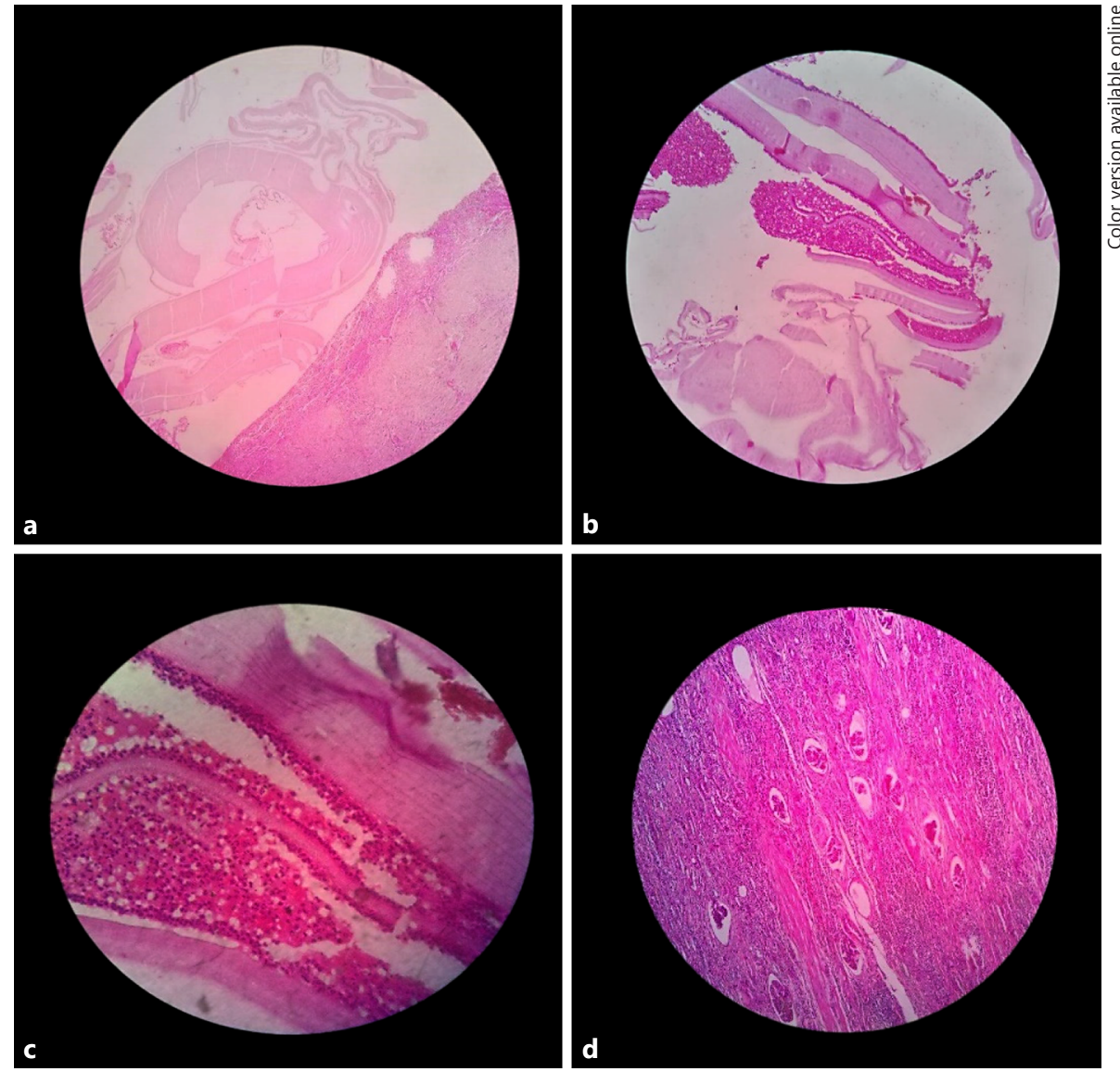
logical picture of a renal hydatid cyst has been described previously as a single unilocular lesion with multiple daughter cysts or vesicles [2]; atypically, daughter cysts may be absent [8]. Similarly, the current case demonstrated only a single unilocular cyst which contributed to the preoperative misdiagnosis of the lesion. An isolated renal hydatid cyst is treated by nephron-sparing surgery in about $75 \%$ of cases $[1,8]$. Nephrectomy is warranted in the case of preoperative undiagnosed masses [2]. The latter scenario occurred in the current case.

\section{Conclusion}

In spite of the characteristic picture of cystic echinococcosis on imaging, an isolated renal hydatid cyst is a very rare lesion which may be misdiagnosed as a renal tumor. This should be taken into consideration in the differential diagnosis of single or multiple renal cystic lesions. It should be considered a tumor until proven otherwise, even in endemic countries for echinococcosis. Nephrectomy is warranted for preoperatively misdiagnosed large lesions and high-grade lesions.

\section{References}

1 Aggarwal S, Bansal A: Laparoscopic management of renal hydatid cyst. J Soc Laparoend 2014; 18:361-366.

12 Qadri S, Sherwani RK, Ahmed M: Isolated cystic echinococcosis of kidney burlesquing as renal cell carcinoma: a diagnostic pitfall. Ann Parasitol 2015;61:57-60.

3 Gupta M, Singh R, Lehl SS: Isolated renal hydatid cyst in a diabetic postmenopausal female. J Midlife Health 2015;6:134-136.
4 Choi H, Park JY, Kim JH, et al: Primary renal hydatid cyst: mis-interpretation as a renal malignancy. Korean J Parasitol 2014;52:295298.

5 World Health Organization Informal Working Group: International classification of ultrasound images in cystic echinococcosis for application in clinical and field epidemiological settings. Acta Trop 2003;85:253-261.

$\checkmark 6$ Brunetti E, Kern P, Vuitton DA: Expert consensus for the diagnosis and treatment of cystic and alveolar echinococcosis in humans. Acta Trop 2010;114:1-16.
7 Stojkovic M, Rosenberger K, Kauczor H-U, et al: Diagnosing and staging of cystic echinococcosis: how do CT and MRI perform in comparison to ultrasound? PLoS Negl Trop Dis 2012;6:e1880.

8 Paramythiotis D, Babgeas P, Kofina K, et al: Presence of an isolated hydatid cyst in the left kidney: report of a case of this rare condition managed surgically. Case Rep Urol 2016;2016: 6902082. 